\title{
Homeomorphisms which are Dehn twists on the boundary
}

\author{
DARRYL MCCULLOUGH
}

\begin{abstract}
A homeomorphism of a 3-manifold $M$ is said to be Dehn twists on the boundary when its restriction to $\partial M$ is isotopic to the identity on the complement of a collection of disjoint simple closed curves in $\partial M$. In this paper, we give various results about such collections of curves and the associated homeomorphisms. In particular, if $M$ is compact, orientable, irreducible and $\partial M$ is a single torus, and $M$ admits a homeomorphism which is a nontrivial Dehn twist on $\partial M$, then $M$ must be a solid torus.
\end{abstract}

57M99; 57R50

\section{Introduction}

A homeomorphism $h$ of a compact 3-manifold $M$ is said to be Dehn twists on the boundary when its restriction to $\partial M$ is isotopic to the identity on the complement of a collection of disjoint simple closed curves in $\partial M$. If this collection is nonempty, and the restricted homeomorphism is not isotopic to the identity on the complement of any proper subset of the collection, then we say that $h$ is Dehn twists about the collection. The restriction of $h$ to $\partial M$ is then isotopic to a composition of nontrivial Dehn twists about the curves, where for us a Dehn twist may be a power of a "single" Dehn twist. Note that the minimality condition implies that each curve in the collection is essential in $\partial M$, and no two of them are isotopic in $\partial M$. Our first main result gives strong restrictions on the collection of curves.

Theorem 1 Let $M$ be a compact orientable 3-manifold which admits a homeomorphism which is Dehn twists on the boundary about the collection $C_{1}, \ldots, C_{n}$ of simple closed curves in $\partial M$. Then for each $i$, either $C_{i}$ bounds a disk in $M$, or for some $j \neq i, C_{i}$ and $C_{j}$ cobound an incompressible annulus in $M$.

Our second main result gives structural information about such homeomorphisms. It refers to Dehn twists about disks and annuli in $M$, whose definition is recalled in Section 1. 
Theorem 2 Let $M$ be a compact orientable 3-manifold which admits a homeomorphism which is Dehn twists on the boundary about the collection $C_{1}, \ldots, C_{n}$ of simple closed curves in $\partial M$. Then there exists a collection of disjoint imbedded disks and annuli in $M$, each of whose boundary circles is isotopic to one of the $C_{i}$, for which some composition of Dehn twists about these disks and annuli is isotopic to $h$ on $\partial M$.

That is, $h$ must arise in the most obvious way, by composition of Dehn twists about a collection of disjoint annuli and disks with a homeomorphism that is the identity on the boundary.

Theorem 1 and Theorem 2 yield strong statements for specific classes of manifolds. For the case when $M$ is a compression body, examined in Section 3, a homeomorphism which is Dehn twists on the boundary is actually isotopic to a product of Dehn twists about disjoint annuli and disks. This appears in Oertel [10] for the case when $M$ is a handlebody. Another application is the following:

Corollary 3 Let $M$ be a compact orientable irreducible 3-manifold with boundary a torus. If $M$ admits a homeomorphism which is a Dehn twist on $\partial M$, then $M$ is a solid torus and the homeomorphism is isotopic to a Dehn twist about a meridian disk.

In particular, the only knot complement in $S^{3}$ (indeed, the only irreducible complement of a knot in any closed orientable 3-manifold) admitting a homeomorphism which is a nontrivial Dehn twist on the boundary is the trivial knot.

Proof of Corollary 3 By Theorem 1, $C$ bounds a disk in $M$. Since $M$ is irreducible, this implies that $M$ is a solid torus. By Theorem 2, there is a Dehn twist about the meridian disk which is isotopic on $\partial M$ to the original homeomorphism, and since any homeomorphism which is the identity on the boundary of a solid torus is isotopic to the identity, this Dehn twist and the original homeomorphism must be isotopic.

It appears that most of our results could be extended to the nonorientable case, adding the possibility of Dehn twists about Möbius bands in Theorem 1, Theorem 2 and Corollary 6 , but the proof of Lemma 5 would require the more elaborate machinery of uniform homeomorphisms, found in McCullough [7] or Chapter 12 of Canary and McCullough [2] (in particular, Lemma 12.1.2 of [2] is a version of Lemma 1.4 of [7] that applies to nonorientable 3-manifolds). Corollary 3 fails in the nonorientable case, however. Not only can a nonorientable manifold with torus boundary admit Dehn twists about Möbius bands, but an annulus can meet the torus boundary in such a way that a Dehn twist about the annulus will be isotopic on the boundary torus to an even power of a simple Dehn twist about one of its boundary circles. 
Some of the work presented here is applied in the article Knot adjacency, genus and essential tori by E Kalfagianni and X-S Lin [5]. We are grateful to the authors of that paper for originally bringing the possibility of results like Theorem 1 and Theorem 2 to our attention.

The research in this article was supported in part by NSF grant DMS-0102463.

\section{Proof of Theorem 1}

Recall that Dehn twists in 3-manifolds can be defined as follows. Consider first a properly imbedded and 2-sided disk or annulus $F$ in a 3-manifold $M$. Imbed the product $F \times[0,1]$ in $M$ so that $(F \times[0,1]) \cap \partial M=\partial F \times[0,1]$ and $F \times\{0\}=F$. Let $r_{\theta}$ rotate $F$ through an angle $\theta$ (that is, if $F$ is a disk, rotate about the origin, and if it is an annulus $S^{1} \times[0,1]$, rotate in the $S^{1}$-factor). Fixing some integer $n$, define $t: M \rightarrow M$ by $t(x)=x$ for $x \notin F \times[0,1]$ and $t(z, s)=\left(r_{2 \pi n s}(z), s\right)$ if $(z, s) \in F \times[0,1]$. The restriction of $t$ to $\partial M$ is a Dehn twist about each circle of $\partial F$. Dehn twists are defined similarly when $F$ is a $2-$ sphere or a two-sided projective plane, Möbius band, torus, or Klein bottle (for the case of tori, there are infinitely many nonisotopic choices of an $S^{1}$-factor to define $r_{\theta}$ ). Since a properly imbedded closed surface in $M$ is disjoint from the boundary, a Dehn twist about a closed surface is the identity on $\partial M$.

The proof of Theorem 1 will use the following result on Dehn twists about annuli in orientable 3-manifolds.

Lemma 4 Let $A_{1}$ and $A_{2}$ be properly imbedded annuli in an orientable 3-manifold $M$, with common boundary consisting of the loops $C^{\prime}$ and $C^{\prime \prime}$. Let $N^{\prime}$ and $N^{\prime \prime}$ be disjoint closed regular neighborhoods in $\partial M$ of $C^{\prime}$ and $C^{\prime \prime}$ respectively, and let $t_{i}$ be Dehn twists about the $A_{i}$ whose restrictions to $\partial M$ are supported on $N^{\prime} \cup N^{\prime \prime}$. If the restrictions of $t_{1}$ and $t_{2}$ to $N^{\prime}$ are isotopic relative to $\partial N^{\prime}$, then their restrictions to $N^{\prime \prime}$ are isotopic relative to $\partial N^{\prime \prime}$. Consequently, if $A$ is a properly imbedded annulus whose boundary circles are isotopic in $\partial M$ (in particular, if they are contained in a torus boundary component of $M$ ), then any Dehn twist about $A$ is isotopic to the identity on $\partial M$.

Proof The result is clear if the $A_{i}$ have orientations so that their induced orientations on $C^{\prime} \cup C^{\prime \prime}$ are equal, since then the imbeddings of $S^{1} \times I \times[0,1]$ into $M$ used to define the Dehn twists can be chosen to agree on $S^{1} \times \partial I \times[0,1]$. So we assume that the oriented boundary of $A_{1}$ is $C^{\prime} \cup C^{\prime \prime}$ and the oriented boundary of $A_{2}$ is $C^{\prime} \cup\left(-C^{\prime \prime}\right)$.

By assumption, $t_{1}$ and $t_{2}$ restrict to the same Dehn twist near $C^{\prime}$. Their effects near $C^{\prime \prime}$ differ in that after cutting along $C^{\prime \prime}$, the twisting of $C^{\prime \prime}$ occurs in opposite directions, 
but since $M$ is orientable, they also differ in that this twisting is extended to collar neighborhoods on opposite sides of $C^{\prime \prime}$ (that is, the imbeddings of $S^{1} \times \partial I \times[0,1]$ used to define the Dehn twists fall on the same side of $C^{\prime}$ but on opposite sides of $\left.C^{\prime \prime}\right)$. Each of these differences changes a Dehn twist about $C^{\prime \prime}$ to its inverse, so their combined effect is to give isotopic Dehn twists near $C^{\prime \prime}$.

The last remark of the lemma follows by taking $A_{1}=A$ and $A_{2}$ to be an annulus with $\partial A_{2}=\partial A_{1}$, with $A_{2}$ parallel into $\partial M$. All Dehn twists about $A_{2}$ are isotopic to the identity on $\partial M$, so the same is true for all Dehn twists about $A_{1}$.

We will also need a fact about homeomorphisms of reducible 3-manifolds, even in many of the cases when $M$ itself is irreducible.

Lemma 5 Let $W=P \# Q$ be a connected sum of compact orientable 3-manifolds, with $P$ irreducible. Let $S$ be the sum 2-sphere. Suppose that $\partial P$ is nonempty and that $g: W \rightarrow W$ is a homeomorphism which preserves a component of $\partial P$. Then there is a homeomorphism $j: W \rightarrow W$, which is the identity on $\partial W$, such that $j g(S)=S$.

Proof Let $P_{1} \# \cdots \# P_{r} \# R_{1} \# \cdots \# R_{S}$ be a prime factorization of $W$, where each $P_{i}$ is irreducible and each $R_{j}$ is $S^{2} \times S^{1}$. Let $\Sigma$ be the result of removing from a 3-sphere the interiors of $r+2 s$ disjoint 3-balls $B_{1}, \ldots, B_{r}, D_{1}, E_{1}, D_{2}, \ldots, E_{s}$. For $1 \leq i \leq r$, let $P_{i}^{\prime}$ be the result of removing the interior of a small open 3-ball $B_{i}^{\prime}$ from $P_{i}$, and regard $W$ as obtained from $\Sigma$ and the union of the $P_{i}^{\prime}$ by identifying each $\partial B_{i}$ with $\partial B_{i}^{\prime}$ and each $\partial D_{j}$ with $\partial E_{j}$.

In [7] and in Section 12.1 of [2], certain slide homeomorphisms of $W$ are constructed. These can be informally described as cutting $W$ apart along a $\partial B_{i}$ or $\partial D_{j}$, filling in one of the removed 3-balls to obtain a manifold $Y$, performing an isotopy that slides that ball around a loop in the interior of $Y$, removing the 3-ball and gluing back together to obtain a homeomorphism of the original $W$. Slide homeomorphisms are assumed to be the identity on $\partial W$ (this is ensured by requiring that the isotopy that slides the 3-ball around the loop in $Y$ be the identity on $\partial Y$ at all times). Lemma 1.4 of [7], essentially due to M Scharlemann, says that if $T$ is a collection of disjoint imbedded 2-spheres in the interior of $W$, then there is a composition $j$ of slide homeomorphisms such that $j(T) \subset \Sigma$.

Since $P$ is irreducible, we may choose notation so that $P=P_{1}$ and $S=\partial B_{1}$. Applying Lemma 1.4 of [7] with $T=g(S)$, we obtain $j$ so that $j g(S) \subset \Sigma$. In particular, there is a component $Z$ of $W-j g(S)$ whose closure contains $P_{1}^{\prime}$. Since $g$ is assumed to preserve a component of $\partial P_{1}$, the closure of $Z$ must be $j g\left(P_{1}^{\prime}\right)$. Since $P_{1}$ is irreducible, $j g(S)$ must be isotopic to $S$ in $W$, so changing $j$ by isotopy we obtain $j g(S)=S$. 
We can now prove Theorem 1 . Let $N_{j}$ be disjoint closed regular neighborhoods of the $C_{j}$ in $\partial M$, and let $F$ be the closure of $\partial M-\cup_{j} N_{j}$. By hypothesis, we may assume that $h$ is the identity on $F$. Let $M^{\prime}$ be another copy of $M$, and identify $F$ with its copy $F^{\prime}$ to form a manifold $W$ with boundary a union of tori, each containing one $C_{j}$. Denote by $T_{j}$ the one containing $C_{j}$. Let $g: W \rightarrow W$ be $h$ on $M$ and the identity map on $M^{\prime}$, so that on each $T_{j}, g$ restricts to a nontrivial Dehn twist about $C_{j}$.

Fix any $C_{i}$, and for notational convenience call it $C_{1}$. Put $W_{1}=W$ if $W$ is irreducible. Otherwise, write $W$ as $W_{1} \# W_{2}$ where $W_{1}$ is irreducible and $T_{1} \subseteq \partial W_{1}$, and let $S$ be the sum sphere. By Lemma 5, there is a homeomorphism $j$ of $W$ that is the identity on $\partial W$, such that $j g(S)=S$. Split $W$ along $S$, fill in one of the resulting 2 -sphere boundary components to obtain $W_{1}$ and extend $j g$ to that ball. This produces a homeomorphism $g_{1}$ of $W_{1}$ that restricts on each boundary torus of $W_{1}$ to a nontrivial Dehn twist about one of the $C_{j}$.

Assume first that $W_{1}$ has compressible boundary. Since $W_{1}$ is irreducible, it is a solid torus with boundary $T_{1}$. The only nontrivial Dehn twists on $T_{1}$ that extend to $W_{1}$ are Dehn twists about a meridian circle, showing that $C_{1}$ bounds a disk in $W_{1}$, and hence a disk $E$ in $W$. Since $C_{1}$ does not meet $F$, we may assume that $E$ meets $F$ transversely in a collection of disjoint circles. The intersection $X^{\prime}$ of $E$ with $M^{\prime}$ has a mirror image $X$ in $M$. Change $E$ by replacing $X^{\prime}$ with $X$, producing a singular disk in $M$ with boundary $C_{1}$. By the Loop Theorem, $C_{1}$ bounds an imbedded disk in $M$.

We call the argument in the previous paragraph that started with $E$ in $W$ and obtained a singular version of $E$ in $M$, having the same boundary as the original $E$, a swapping argument (since we are swapping pieces of the surface on one side of $F$ for pieces on the other side).

Suppose now that $W_{1}$ has incompressible boundary. Let $V_{1}$ be Johannson's characteristic submanifold of $W_{1}$ ([4], also see Chapter 2 of [2] for an exposition of Johannson's theory). Since $\partial W_{1}$ consists of tori, $V_{1}$ admits a Seifert fibering and contains all of $\partial W_{1}$ (in Johannson's definition, a component of $V_{1}$ can be just a collar neighborhood of a torus boundary component). Each $C_{j}$ in $W_{1}$ is noncontractible in $T_{j}$, and $T_{j}$ is incompressible in $W_{1}$, so $C_{j}$ is noncontractible in $W_{1}$. This implies that $C_{j}$ is noncontractible in $W$, hence also in $M$.

It suffices to prove that $C_{1}$ and some other $C_{i}$ cobound an imbedded annulus $A$ in $W_{1}$ and hence in $W$. For then, a swapping argument produces a singular annulus in $M$ cobounded by $C_{1}$ and $C_{i}$. Since $C_{1}$ and $C_{i}$ are noncontractible, a direct application of the Generalized Loop Theorem [11] (see [3, p. 55]) produces an imbedded annulus in $M$ cobounded by $C_{1}$ and $C_{i}$. 
By Corollary 27.6 of [4], the mapping class group of $W_{1}$ contains a subgroup of finite index generated by Dehn twists about essential annuli and tori. So by raising $g_{1}$ to a power, we may assume that it is a composition of such Dehn twists. The Dehn twists about tori do not affect $\partial W_{1}$, so we may discard them to assume that $g_{1}$ is a composition $t_{1} \cdots t_{m}$, where each $t_{k}$ is a Dehn twist about an essential annulus $A_{k}$. By Corollary 10.10 of [4], each $A_{k}$ is isotopic into $V_{1}$. By Proposition 5.6 of [4], we may further change each $A_{k}$ by isotopy to be either horizontal or vertical with respect to the Seifert fibering of $V_{1}$.

Suppose first that some $A_{k}$ is horizontal. Then $V_{1}$ is either $S^{1} \times S^{1} \times I$ or the twisted $I$-bundle over the Klein bottle (a horizontal annulus projects by an orbifold covering map to the base orbifold, and the orbifold Euler characteristic shows that the base orbifold is either an annulus, a Möbius band, or the disk with two order-2 cone points, the latter two possibilities yielding the two Seifert fiberings of the twisted $I$-bundle over the Klein bottle). In the latter case, $\partial V_{1}=T_{1}$, so $W_{1}=V_{1}$ and therefore $\partial W_{1}=T_{1}$. By Lemma 4, each $t_{k}$ is isotopic to the identity on $T_{1}$, hence so is $g_{1}$, a contradiction. So $V_{1}=S^{1} \times S^{1} \times I$.

Since $A_{k}$ is horizontal, it must meet both components of $\partial V_{1}$, and we have $V_{1}=W_{1}$ and $\partial W_{1}=T_{1} \cup T_{i}$ for some $i$. Let $A_{0}=C_{1} \times I \subset S^{1} \times S^{1} \times I$. For an appropriate Dehn twist $t$ about $A_{0}, t^{-1} g$ is isotopic to the identity on $T_{1}$. Using Lemma 3.5 of [12], $t^{-1} g$ is isotopic to a level-preserving homeomorphism of $W_{1}$, and hence to the identity. We conclude that $g_{1}$ is isotopic to $t$, and consequently $C_{1}$ and $C_{i}$ cobound an annulus in $W_{1}$.

It remains to consider the case when all $A_{k}$ are vertical. In this case, each $t_{k}$ restricts on $\partial W_{1}$ to Dehn twists about loops isotopic to fibers, so each $C_{j}$ in $\partial W_{1}$ is isotopic to a fiber of the Seifert fibering on $V_{1}$.

Let $V_{1}^{\prime}$ be the component of $V_{1}$ that contains $C_{1}$. Suppose first that $V_{1}^{\prime} \cap \partial W_{1}=T_{1}$. Then each $A_{k}$ that meets $T_{1}$ has both boundary circles in $T_{1}$, so Lemma 4 implies that $g_{1}$ is isotopic to the identity on $T_{1}$, a contradiction. So $V_{1}^{\prime}$ contains another $T_{i}$. Since $C_{1}$ and $C_{i}$ are isotopic to fibers, there is an annulus in $V_{1}^{\prime}$ with boundary $C_{1} \cup C_{i}$.

\section{Proof of Theorem 2}

Theorem 1 provides a properly imbedded surface $S$ which is either an imbedded disk with boundary $C_{n}$ or an incompressible annulus with boundary $C_{n}$ and some other $C_{i}$. For some Dehn twist $t_{n}$ about $S, t_{n}$ and $h$ are isotopic near $C_{n}$. The composition $t_{n}^{-1} h$ is isotopic on $\partial M$ to a composition of Dehn twists about $C_{1}, \ldots, C_{n-1}$ (some 
of them possibly trivial). Induction on $n$ produces a composition $t$ as in the theorem, except for the assertion that the disks and annuli may be selected to be disjoint.

Let $D_{1}, \ldots, D_{r}$ and $A_{1}, \ldots, A_{s}$ be the disks and annuli needed for the Dehn twists in $t$. We first work on the annuli.

We will say that a union $\mathcal{A}$ of disjoint incompressible imbedded annuli in $M$ is sufficient for $A_{1}, \ldots, A_{k}$ if each boundary circle of $\mathcal{A}$ is isotopic in $\partial M$ to a boundary circle of one of the $A_{i}$, and if for any composition of Dehn twists about the set $\left(\cup_{i=1}^{r} D_{i}\right) \cup\left(\cup_{j=1}^{k} A_{j}\right)$, there is a composition of Dehn twists about the union of $\cup_{i=1}^{r} D_{i}$ and the annuli of $\mathcal{A}$ which has the same effect, up to isotopy, on $\partial M$. In particular, $\mathcal{A}=A_{1}$ is sufficient for $A_{1}$ alone. Inductively, suppose that $\mathcal{A}$ is sufficient for $A_{1}, \ldots, A_{k-1}$. By a routine surgery process, we may change $\mathcal{A}$ so that $A_{k}$ and $\mathcal{A}$ intersect only in circles essential in both $A_{k}$ and $\mathcal{A}$. (First, make $\mathcal{A}$ transverse to $A_{k}$. An intersection circle which is contractible in $\mathcal{A}$ must also be contractible in $A_{k}$, since both $\mathcal{A}$ and $A_{k}$ are incompressible. If there is a contractible intersection circle, then there is a disk $E$ in $A_{k}$ with $\partial E$ a component of $A_{k} \cap \mathcal{A}$ and the interior of $E$ disjoint from $\mathcal{A}$. Replace the disk in $\mathcal{A}$ bounded by $\partial E$ with $E$, and push off by isotopy to achieve a reduction of $A_{k} \cap \mathcal{A}$.)

Now let $Z$ be a closed regular neighborhood of $A_{k} \cup \mathcal{A}$. Since all intersection circles of $A_{k}$ with $\mathcal{A}$ are essential in both intersecting annuli, each component of $Z$ has a structure as an $S^{1}$-bundle in which the boundary circles of $\mathcal{A}$ and $A_{k}$ are fibers.

We will show that $Z$ contains a collection sufficient for $A_{k} \cup \mathcal{A}$ and hence also for $A_{1}, \ldots, A_{k}$. We may assume that $Z$ is connected. For notational simplicity, there is no harm in writing $C_{1}, \ldots, C_{m}$ for the boundary circles of $\mathcal{A}$ and $A_{k}$, since they are isotopic in $\partial M$ to some of the original $C_{i}$.

Fix a small annular neighborhood $N$ of $C_{1}$ in $Z \cap \partial M$. Using the $S^{1}$-bundle structure of $Z$, we can choose a collection $B_{2}, \ldots, B_{m}$ of disjoint annuli, with $B_{i}$ running from $C_{i}$ to a loop in $N$ parallel to $C_{1}$.

Consider one of the annuli $A$ of $A_{k} \cup \mathcal{A}$, say with boundary circles isotopic to $C_{i}$ and $C_{j}$. If either $i$ or $j$ is 1 , say $j=1$, then by Lemma 4, Dehn twists about $A$ have the same effect on $\partial M$ as Dehn twists about $B_{i}$. If neither is 1 , form an annulus $B$ connecting $C_{i}$ to $C_{j}$ by taking the union of $B_{i}, B_{j}$, and the annulus in $N$ connecting $B_{i} \cap N$ to $B_{j} \cap N$, then pushing off of $N$ to obtain a properly imbedded annulus. Observe that any Dehn twist about $B$ is isotopic on $M$ to a composition of Dehn twists about $B_{i}$ and $B_{j}$. By Lemma 4 , there is a Dehn twist about $B$ whose effect on $\partial M$ is the same as the twist about $A$. This shows that the collection $B_{2}, \ldots, B_{m}$ is sufficient for $A_{1}, \ldots, A_{k}$ and completes the induction. So there is a collection $\mathcal{A}$ sufficient for $A_{1}, \ldots, A_{s}$. 
By further routine surgery, we may assume that each $D_{i}$ is disjoint from $\mathcal{A}$. Then, surger $D_{2}$ to make $D_{2}$ disjoint from $D_{1}$, surger $D_{3}$ to make it disjoint from $D_{1} \cup D_{2}$, and so on, eventually achieving the desired collection of disjoint disks and annuli.

\section{Compression bodies}

Compression bodies were developed by F Bonahon [1], in a study of cobordism of surface homeomorphisms. They were used in work on mapping class groups of 3manifolds $[9 ; 6 ; 8]$ and on deformations of hyperbolic structures on 3-manifolds [2]. The homeomorphisms of compression bodies were further investigated by Oertel [10], who develops an analogue for compression bodies of the Nielsen-Thurston theory of surface homeomorphisms.

To fix notation and terminology, we recall that a compression body is a connected 3-manifold $V$ constructed by starting with a compact surface $G$ with no components that are 2 -spheres, forming $G \times[0,1]$, and then attaching 1 -handles to $G \times\{1\}$. Compression bodies are irreducible. They can be handlebodies (when no component of $G$ is closed) or product $I$-bundles (when there are no 1 -handles). The exterior boundary of $V$ is $\partial V-(G \times\{0\} \cup \partial G \times[0,1))$. Note that if $F$ is the exterior boundary of $V$, and $N$ is a (small) regular neighborhood in $V$ of the union of $F$ with a collection of cocore 2-disks for the 1-handles of $V$, then each component of $\overline{V-N}$ is a product $X \times I$, where $X \times\{0\}$ is a component of the frontier of $N$ and $X \times\{1\}$ is a component of $G \times\{0\}$.

The following result was proven in [10] for the case of $V$ a handlebody.

Corollary 6 Let $V$ be a compact orientable compression body, and let $h: V \rightarrow V$ be a homeomorphism which is Dehn twists on the boundary about the collection $C_{1}, \ldots$, $C_{n}$ of simple closed curves in $\partial V$. Then $h$ is isotopic to a composition of Dehn twists about a collection of disjoint disks and incompressible annuli in $V$, each of whose boundary circles is isotopic in $\partial V$ to one of the $C_{i}$.

To prove Corollary 6, we note first that by Theorem 2, there is a composition $t$ of Dehn twists about a collection of disjoint disks and incompressible annuli in $V$, such that $t$ and $h$ are isotopic on $\partial V$. Changing $h$ by isotopy, we may assume that $t^{-1} h$ is the identity on $\partial V$. Corollary 6 is then immediate from the following lemma.

Lemma 7 Let $V$ be a compression body with exterior boundary $F$, and let $g: V \rightarrow V$ be a homeomorphism which is the identity on $F$. Then $g$ is isotopic relative to $F$ to the identity. 
Proof We have noted that there is a collection of disjoint properly imbedded disks $E_{1}, \ldots, E_{n}$, with boundaries in $F$, such that if $N$ is a regular neighborhood of $F \cup\left(\cup_{i} E_{i}\right)$, then each component of $\overline{V-N}$ is a product $X \times I$, where $X \times\{0\}$ is a component of the frontier of $N$. Now $\partial E_{1}$ is fixed by $g$, so we may assume that $g\left(E_{1}\right) \cap E_{1}$ consists of $\partial E_{1}$ and a collection of transverse intersection circles. Since $V$ is irreducible, we may change $g$ by isotopy relative to $F$ to eliminate these other intersection circles, and finally to make $g$ fix $E_{1}$ as well as $F$. Inductively, we may assume that $g$ is the identity on $F \cup\left(\cup_{i} E_{i}\right)$ and then on $N$. Finally, for each component $X \times I$ of $\overline{V-N}, g$ is the identity on $X \times\{0\}$. Using Lemma 3.5 of [12], $g$ may be assumed to preserve the levels $X \times\{s\}$ of $X \times I$, and then there is an obvious isotopy from $g$ to the identity on $X \times I$, relative to $X \times\{0\}$. Applying these isotopies on the complementary components of $N$, we make $g$ the identity on $V$.

\section{References}

[1] F Bonahon, Cobordism of automorphisms of surfaces, Ann. Sci. École Norm. Sup. (4) 16 (1983) 237-270 MR732345

[2] R D Canary, D McCullough, Homotopy equivalences of 3-manifolds and deformation theory of Kleinian groups, Mem. Amer. Math. Soc. 172 (2004) xii+218 MR2096234

[3] J Hempel, 3-Manifolds, Princeton University Press, Princeton, NJ (1976) MR0415619

[4] K Johannson, Homotopy equivalences of 3-manifolds with boundaries, Lecture Notes in Mathematics 761, Springer, Berlin (1979) MR551744

[5] E Kalfagianni, X S Lin, Knot adjacency, genus and essential tori, to appear in Pacific J. Math. arXiv:math.GT/0403024

[6] D McCullough, Twist groups of compact 3-manifolds, Topology 24 (1985) 461-474 MR816525

[7] D McCullough, Topological and algebraic automorphisms of 3-manifolds, from: "Groups of self-equivalences and related topics (Montreal, PQ, 1988)", Lecture Notes in Math. 1425, Springer, Berlin (1990) 102-113 MR1070580

[8] D McCullough, Virtually geometrically finite mapping class groups of 3-manifolds, J. Differential Geom. 33 (1991) 1-65 MR1085134

[9] D McCullough, A Miller, Homeomorphisms of 3-manifolds with compressible boundary, Mem. Amer. Math. Soc. 61 (1986) xii+100 MR840832

[10] U Oertel, Automorphisms of three-dimensional handlebodies, Topology 41 (2002) 363-410 MR1876895

[11] F Waldhausen, Eine Verallgemeinerung des Schleifensatzes, Topology 6 (1967) 501504 MR0220300 
[12] F Waldhausen, On irreducible 3-manifolds which are sufficiently large, Ann. of Math. (2) 87 (1968) 56-88 MR0224099

Department of Mathematics, University of Oklahoma

Norman, Oklahoma 73019, USA

dmccullough@math.ou. edu

http://www.math.ou.edu/ dmccullough/

Received: 31 August 2005 Revised: 6 July 2006

Algebraic 83 Geometric Topology, Volume 6 (2006) 